\title{
New CT signs of alveolar cell carcinoma
}

L D R Tsatsi
MB ChB, FC Rad (D) SA
J O Ozoh
BSc, MB BS, FMCR (Rad) Nigeria
Department of Diagnostic Radiology
Medical University of Southern Africa

\section{Case presentation}

An 83-year-old female patient was referred to the Medical Outpatient Department of Ga-Rankuwa Hospital in March 2002 by a general practitioner. Her complaint was chest pain and a productive cough with bloodstreaked white sputum of one-month duration. Her past medical history appeared uneventful. She was a nonsmoker. Her medication history revealed that she has been on antibiotics non-stop for more than one month. On examination her respiration was $34 / \mathrm{min}$, temperature $38^{\circ} \mathrm{C}$, pulse 90 beats/min and blood pressure $110 / 80 \mathrm{mmHg}$. There was no lymphadenopathy; however, an incidental umbilical hernia was found. An auscultation widespread agitation was found and she coughed out white frothy sputum during the examination. A provisional diagnosis of intractable pneumonia was made.

Acid-fast bacilli tests were negative on three consecutive occasions.

A lung function test revealed a restrictive pattern.

\section{Radiological investigations}

\section{Chest radiography}

- Left lower lobe opacification (Figs 1 and 2)

- Air bronchiograms in the left lower lobe (Figs 3 and 4)

- No lymphadenopathy (Figs 1 - 4)

- No signs of lobar collapse (Figs 1 and 2)

- Cardiomediastinal relationships normal.

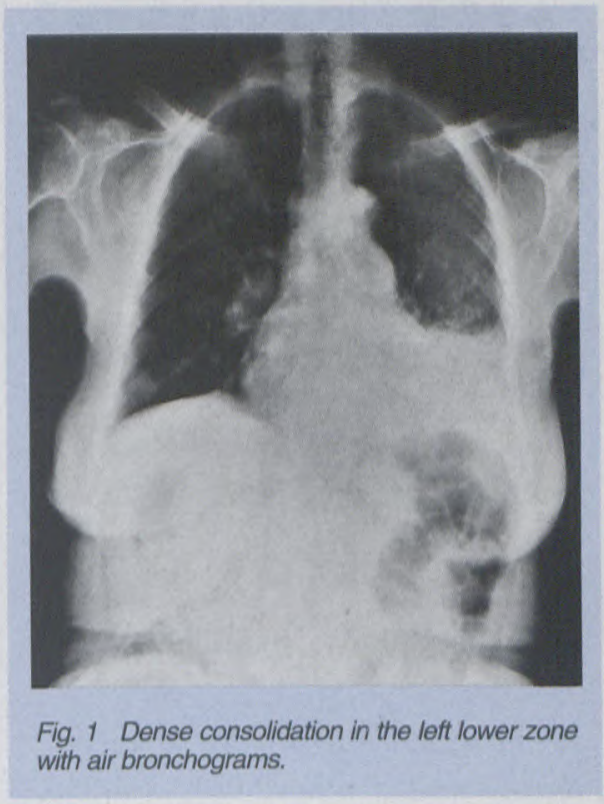

\section{CT scan}

One hundred millilitres of intravenous iopromide was used $(300 \mathrm{mg}$, Schering, Berlin, Germany) for contrast studies of the chest. Non-contrasted studies were also performed. Chest CT photographed on both lung
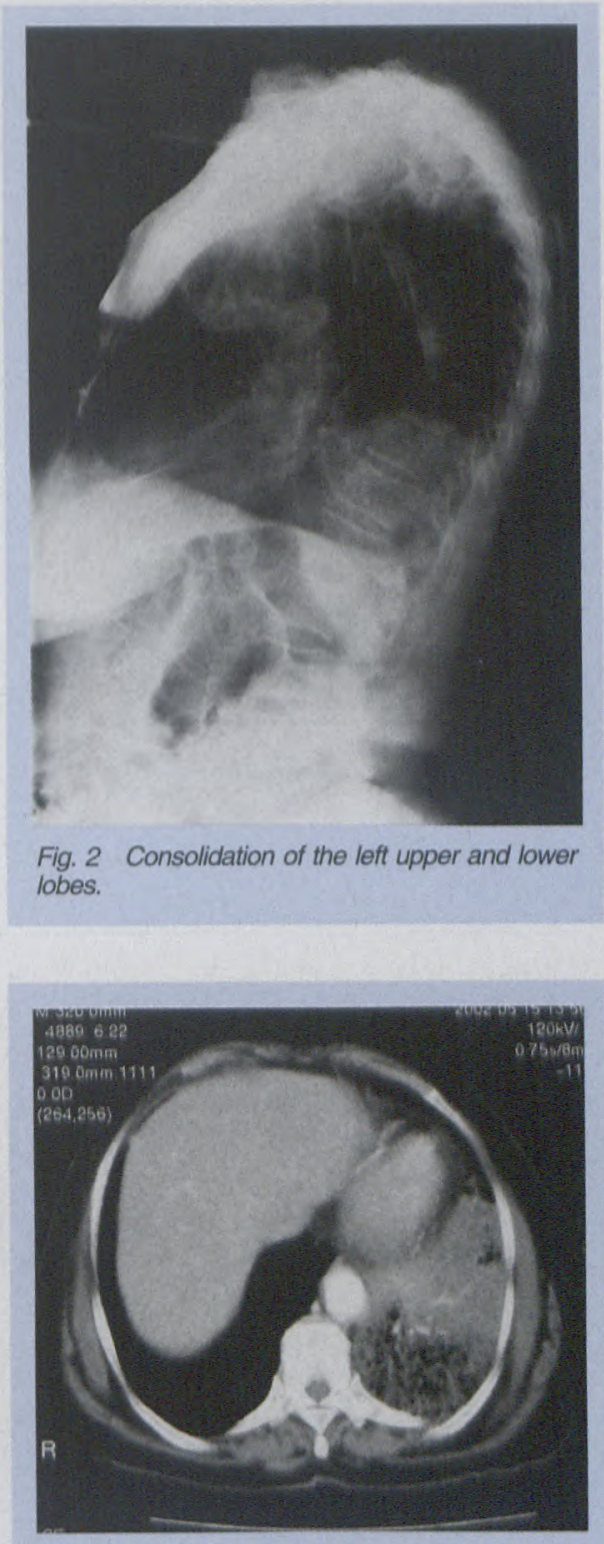

Fig. 3. Post-contrast axial CT showing an angiogram sign and patchy consolidation.

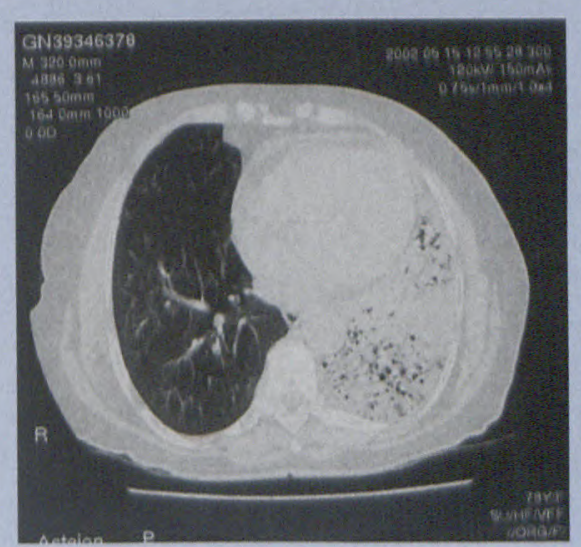

Fig. 4 Axial CT showing squeezing and bending of air bronchograms. 


\section{CASE REPORT}

and mediastinal windows demonstrated the CT angiogram sign. Attenuation of the lobe was heterogeneous. ${ }^{1}$ Multiple air bronchograms were seen ${ }^{1,2}$ There was dilatation, stretching, sweeping, widening of the angle and crowding of bronchi .

A pleural effusion was noted.

\section{Discussion}

Our patient had specific signs of bronchoalveolar consolidation which include squeezing, stretching and sweeping patent air bronchograms within the consolidated lung. ${ }^{3}$ The 'crazy paving' pattern is due to thick- ening of the interlobular septae. ${ }^{3}$ All these changes, due to unique lepidic growth of the tumour were also noted. ${ }^{4}$

Our patient did not have satellite lesions or bulging tissues, which increase the likelihood of bronchoalveolar carcinoma (BAC). Other absent signs were pseudocavitation, air fluid level in cavities and marginal enhancement. ${ }^{4.5}$

Although the CT angiogram sign was present it is also seen in pneumo$\mathrm{nia}$, and is therefore nonspecific. ${ }^{4-6}$

\section{References}

1. Akata S, Fukushima A, Kakizaki D, Ase K, Amino S. CT scanning of bronchioalveolar carcinoma: specific appearances. Lung Cancer 1995; 12: 221-230.

2. Im J, Han MC, Yu EJ, et al. Lobar bronchioalveolar carcinoma: 'angiogram sign' on CT scans. Radiology 1990; 176: 749-753.

3. Zeuthlin N, Lasser EC, Rigler LG. Bronchographic abnormalities in alveolar cell carcinoma of the lung. Dis Chest 1954; 25: 542 549.

4. Im J, Cho Bl, Park JH, et al. CT findings of lobar bronchioalveolar carcinoma. J Comput Assist Tomogr 1986; 10: 320-322.

5. Manning JT Jr, Spjut HJ, Tschen JA. Bronchioloalveolar carcinoma: the significance of histopathologic types. Cancer 1984; 54: 525534.

6. Barsky SH, Grossman DA, Ho J, Holmes EC The multifocality of bronchioloalveolar lung carcinoma: evidence and implications of a multiclonal origin. Mod Pathol 1994; 7: 633-640.

\section{Occult spinal dysraphism}

\section{D R Tsatsi MB ChB, FCRad D)SA}

Department of Diagnostic Radiology Medical University of Southerm Africa

\section{B Okoli} MB BS, MMed

Department of Neurosurgery Medical University of Southern Africa

\section{Case presentation}

A 2-month-old male patient presented to our outpatient's department with a diffuse back swelling. The child is the fifth in a family with no history of congenital abnormalities.

The pregnancy went full term and was a normal vaginal delivery.

A diffuse swelling was noted in the midline in the lumbar region. The mass was covered with normal skin, with no discolouration, hair, sinus or ulceration. It had a soft, fatty feel on palpation.

There was no neurological dysfunction.

Plain film X-rays of the spine demonstrated spina bifida involving the whole spine with sparing of only T12, L1, L2 and L3 (Figs 1 and 2).

The defects were more pronounced in the upper cervical and sacral areas. The spinous processes in the thoracic and lumbar areas were visualised though they were not fused. A CT scan reconstruction of the

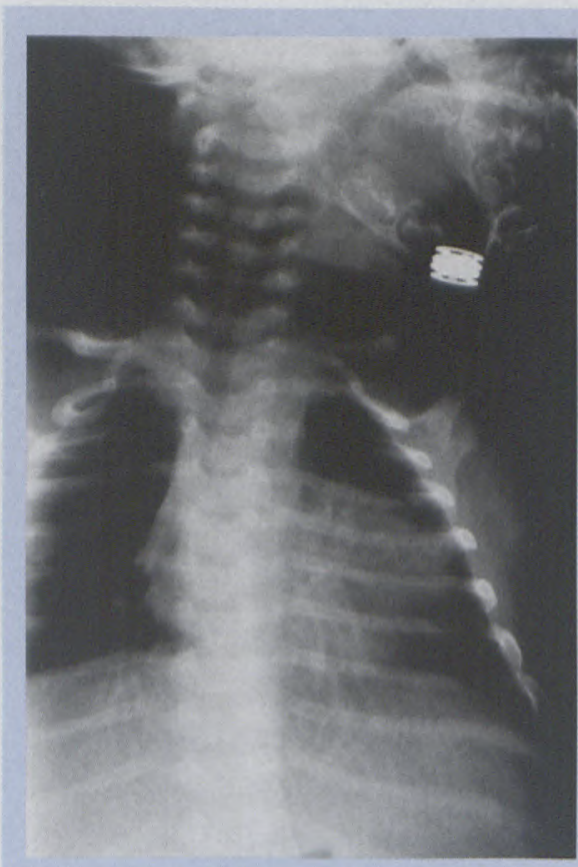

Fig. 1 AP spinal X-ray of the cervical and thoracic spine showing the extensive spina bifida involving both regions.

whole spine demonstrated the spina bifida (Figs 3 and 4 ).

MRI findings showed a normal cord from the cervical to the sacral level. 